\title{
INPUT MODELING FOR HOSPITAL SIMULATION MODELS USING ELECTRONIC MESSAGES
}

\author{
Renata A. Konrad \\ Department of Management \\ Worcester Polytechnic Institute \\ Worcester, MA 01609, USA
}

\author{
Mark A. Lawley \\ School of Biomechanical Engineering \\ Purdue University \\ West Lafayette, IN 47907, USA
}

\begin{abstract}
Health care organizations function in a complex, non-integrated setting, yet the coordination of information, tasks, and equipment across multiple units is essential for productive operations. A variety of simulation models of hospitals exist; however, few reflect resource sharing across multiple departments. Furthermore few models capture the inherent heterogeneity of a hospital's patient mix which plays a crucial role in determining how care is delivered and resources allocated. Patient flow paths can be used as input data to provide systematic insight into resource allocation processes and medical care within a hospital. To date, flow path approaches to studying hospital operations have been hindered by lack of a comprehensive data source. This tutorial describes how electronic communication exchanges between hospital departments are used to create an input model for hospital simulations.
\end{abstract}

\section{INTRODUCTION}

Most hospitals manage their operations with rudimentary census snapshot reports along with ad-hoc use of data from multiple sources to augment their managerial intuition. (Isken 2002) In this era of fast and inexpensive computing, more sophisticated methods are feasible. Similar operational decisions and quality initiatives have been successfully addressed in other industries, yet hospital managers rarely have access to the sophisticated decision support systems common to other industries. (Reid et al. 2005)

Researchers contend that a patient flow approach has greater potential to satisfy the informational needs of health care managers than existing approaches. (Hughes et al. 1998) Patient flow is the progression of activities a patient undergoes through the hospital. Flow information provides better matching of hospital resources to fluctuating demand levels; reduces ambulance diversions (Cameron, Scown, and Campbell 2002; Fatovich, Nagree, and Sprivulis 2005; Shull et al 2003); reduces periods of under and overstaffing (Ledersnaider and Channon 1998), and manages inpatient bed capacity (Mackay 2001).

Patient flow modeling is challenging on account of three factors: lack of consideration of patient activities from admission to discharge, incomplete acknowledgement of patient heterogeneity, and poor input data.

Hospital departments, such as radiology or the emergency department, do not exist in isolation. Resource availability, allocation, and usage in one department impacts resources in another. Many existing analyses of hospitals fail to consider these dependencies thus rarely considering the "total care chain" from admission to discharge. Rather, existent models tend to focus on the analysis of individual units. (Jun, Jacobson, and Swisher 1999) By ignoring the multiple interacting flows of people, materials, and equipment between departments, an analysis risks incomplete portrayal of a hospital's environment. (Marshall, Vasilakis, and El-Darzi 2005) Not surprisingly, suggested solutions from these incomplete models are problematic. (Harper 2002; Marshall, Vasilakis, and El-Darzi 2005; Smith and Goddard 2002)

Likewise, the inherent variability in patient diagnoses has a major impact on analysis of day-to-day hospital operations and is a primary driver of resource use in hospitals. Appropriate nurse staffing levels are directly affected by the types of patients in a hospital along with the level of care required by each patient. A patient mix also generates demand for a myriad of auxiliary services including laboratory, pharmacy, therapy, radiology, housekeeping, and surgical services.

Analyses of hospital operations are also plagued by the lack of comprehensive and sufficiently detailed data. The level of detail required to describe patient flow can be overwhelming - thousands of possible patient paths, hundreds of procedures with varying durations, and the problem of amassing and storing vast amounts of data. Some researchers and analysts attempt to address system complexity by sacrificing resolution. (Harper 2002) Others resort to expensive, setting-specific manual da- 


\section{Konrad and Lawley}

ta collection.(Brahimi and Worthington 1991, Vanden Bosch and Dietz 2000, Swisher and Jacobson 2002). Swisher and Jacobson (2002) discuss numerous time and budgetary constraints associated with collecting data regarding patient flow patterns and task durations. Even if manual data collection is successful, the sample size is often small (Brahimi and Worthington 1991), and researchers are limited to a scant number of patient diagnosis often restricted to a single hospital unit. Current data sources for hospital analyses are expensive, have limited transferability, or insufficiently describe the true hospital population.

Electronic data sources offer an inexpensive way to rapidly collect vast amounts of patient activity data required for analysis. Nonetheless, the industry currently lacks a good source of electronic patient flow data. Healthcare information systems are fraught with integration, interoperability, and infrastructure issues among the various information technologies. (Reid et al. 2005) Typically information systems do not capture data related to the detailed sequence of activities a patient follows in the hospital, making it difficult to characterize patient flow patterns and estimate various simulation input parameters. Electronic medical records present an opportunity to collect detailed flow data; however, electronic medical records are largely in their infancy with low adoption rates. (DesRoches et al. 2008)

The need for comprehensive patient flow input data is evident, yet efforts to date have been hindered by the lack of adequate data acknowledging the impact of patient diagnosis across all hospital units. Information systems messages offer promise in addressing this problem. Much of what happens to a patient is initiated by, and recorded through, electronic message exchanges between hospital units. Hospital department information systems generate messages regarding medication orders, lab requests, and transfer orders among many other activities. Collectively these messages provide a comprehensive source of data for patient flow. To ensure system interoperability, the messages are presented in a standardized format called Health Level Seven or HL7. (Health Level Seven n.d.) This messaging standard is widely used across the healthcare industry enabling disparate healthcare applications to exchange sets of clinical and administrative data. (Health Level Seven n.d.)

Information system trace logs contain HL7 formatted messages with time-stamped data representing much of what happens during a patient's stay. These messages contain details about the status of physician orders and results for labs, procedures and diagnostic tests. Vital signs and procedure notes can also be found. In addition to clinical information, messages sent between information systems record bed transfers, a patient's location, or the time of discharge.

Information system messaging is a ubiquitous, highly structured, and electronic data source for reconstructing a patient's path by diagnosis. These communication exchanges are found in all hospitals irrespective of whether the organization uses electronic medical records. As such, a method to reconstruct patient flow from this data source is transferable between institutions.

This tutorial illustrates how HL7 formatted information system messages can be used to reconstruct patient hospital experiences by diagnosis. Patient flow is the set of activities a patient undergoes and resources they use throughout their entire hospital stay including auxiliary services and different medical departments. This definition acknowledges that patient activity in one department ultimately is impacted by, and impacts, patient activity in others.

This tutorial bridges the gap that exists in the acquisition and processing of large volumes of very detailed patient flow data necessary for effective simulation analysis of hospital operations (such as admission profiles and bed capacity decisions). The main contribution of this research is the development of an overall approach for data modeling of information system messages data that captures the resource requirements of patients throughout various hospital departments.

The remainder of the tutorial is structured as following. The following section describes the role of simulation in hospital operational analysis. A survey of existing electronic data sources for hospital simulation models is presented. We found that existing data sources do not sufficiently meet modeling needs. In Section 3, we describe an alternative source of data- HL7 formatted information system messages which are effective in modeling patient flow by diagnosis. Section 4 discusses how inpatient flow models are constructed. The following section introduces how this type of data model can enhance a simulation model examining short-term operational decisions. Section 6 summarizes the key points of the tutorial paper.

\section{BACKGROUND}

\subsection{Simulation Models of Hospital Operations}

Discrete event simulation (DES) is a widely used method for analyzing hospital operations. Given the complex relationships which exist in hospital processes, simulation is typically favored over analytic approximations. Ten years ago, Jun, Jacobson, and Swisher (1999) surveyed almost 120 works in this area reflecting the popularity of this approach. A recent extensive overview of the use of DES in healthcare research can be found in Jacobson, Hall, and Swisher (2006).

Existing models primarily focus on analysing and improving patient throughput, and allocating resources by considering staffing requirements, staff utilization or bed allocation. Using simulation to analyze patient throughput by looking at their length of stay is well documented. For example Oddoye et al. (2009) used a simulation and goal programming approach to 


\section{Konrad and Lawley}

test different staffing scenarios with the objective of improving clinical workflow through a reduction in the patient length of stay in a medical assessment unit. Kolker (2008) used simulation to establish the relationship between ambulance diversions and the patient's length of stay in the Emergency Department. Kolker found that if those patients admitted through the emergency department stay less than 6 hours in the emergency department, the department is on ambulance diversion less than $0.5 \%$ of the time. A large number of simulation efforts to improve patient throughput focus on various scheduling problems. Recent examples of patient scheduling include (Wijewickrama and Takakuwa 2006, Vermeulen et al. 2009) staff scheduling (Hung et al. 2007; Spry and Lawley 2005), and operating room scheduling (Klassen and Rohleder 1996; Denton et al. 2006). Other insightful examples of throughput analysis include determining causes of Emergency Department patient wait times (Takakuwa and Shiozaki 2004); operating theatre allocation (Komashie, Mousavi, and Gore 2008) or facility layout (Sepulveda et al. 1999).

The other predominant focus of simulation models are tactical and strategic decision making such as determining the number of beds needed in a unit or the ideal number of staff. For example, Baesler, Jahnsen, and DaCosta (2003) estimate of the maximum patient load in a Chilean Emergency Department and determine the resources required to care for these patients. VanBerkel and Blake (2007) develop a simulation model to examine capacity planning decisions and to analyze the performance of a general surgery unit for a health region. The study goal was to determine how to redistribute beds between sites to achieve a standard patient length of stay.

This brief literature review conveys the significant amount of research undertaken in simulation applications to patient throughput and resource allocation. The studies reported in the literature have greatly advanced the understanding of the relationship between inputs into the healthcare system and its output performance measures. However, the majority of these studies focus on a specific class of patients or on an individual hospital department. Lowery and Martin (1992) and Jun, Jacobson, and Swisher (1999) suggest the need to develop a comprehensive modeling framework capable of capturing interdepartmental resource relationships.

For a systematic, hospital-wide analysis the following factors must be acknowledged: (1) that resources across the entire hospital are coordinated, and (2) that a hospital serves a broad patient mix. As discussed in the Introduction of this paper, a patient flow approach enables this comprehensive perspective.

\subsection{Electronic Data Sources for Patient Flow Data}

Numerous studies allude to the difficulty of collecting patient flow data though these are mainly in the outpatient setting. (Rajagopalan and Isken 2001) Because of the complexity of various patient routings, many researchers must resort to manually collecting patient flow data (see (Vanden Bosch and Dietz 2000) ). Brahimi and Worthington (1991) mention how both researchers and nursing staff manually collected flow data, gathering only 114 samples without differentiating by diagnoses. If patient flow is to be used as means to analyze hospital system issues, time-consuming manual data collection techniques are completely ineffective.

Electronic data sources offer an opportunity to rapidly collect vast amounts of patient activity data as required by flow models. Unfortunately, the industry currently lacks a good source of electronic patient flow data. Healthcare information systems are fraught with integration, interoperability, and infrastructure issues among the various information technologies. (Reid et al. 2005) A comprehensive data source of patient flow across multiple departments is still non-existent.

Isken et al. (2005) develop a method to automatically characterize outpatient flow from sensor network data. Sensors collected patient location information on various stops visited throughout the outpatient visit: exam room, waiting area, nursing station, and so forth. The study objectives were to propose a data preparation framework capable of analyzing sensor and staff movement data and then develop data constructs representing patient flow patterns. This research drew on the properties of regular expressions (Friedl 2002) to analyze 2,430 unique outpatient paths from 3,737 patient visits. After data preprocessing and reducing the path by removing consecutive stops, the authors were able to describe the frequency of distinct paths. Despite the advancements in automatically generating patient paths, this study did not account for patient diagnosis.

Lin, Hsieh, and Pan (2005) propose a method of using Hidden Markov Models (HMM) to discover clinical pathways for Normal Spontaneous Delivery patients. Their research proposes a methodology to automatically generate pathways from clinical data although the exact source or format of the data is not explicitly mentioned. The authors use 250 patient records to train the HMMs with four states and then use 29 records to test the generated models. While their research is promising in the successful development of a systematic process to generate clinical paths from data, they approach the problem from a clinical not operational perspective. Their motivation lies in enhancing learning for clinicians and not in establishing tools for operational decision making. As with the study above, the authors do not distinguish between different patient diagnoses.

Ceglowski et al. (2005) illustrate how data mining of patient treatment activities produces a process model of emergency department operations. By extracting over 43,000 records from the emergency department's workflow software database, the 


\section{Konrad and Lawley}

authors describe how process-based self-organizing map analysis yields seven clusters of the most common sets of procedures in the department. The authors claim that using process-based clusters facilitates resource planning.

Likewise, Rajagopalan, and Isken (2001) apply clustering methods to depict patient flow while considering a patient's diagnosis. Although the thrust of their work was assessing of the impact of data preparation processes on data mining, they applied their methods to obstetrical and gynecological patient flow. The data, which originated from several different hospital information systems, was warehoused in a data mart designed specifically to support hospital operations analysis. From this data mart, the authors created a data set of 8,261 records of obstetrical and gynecological patients. The authors had three stages of data preparation- the most basic contained a list of locations visited by a patient during their visit (in essence a flow path), the second added external patient classifications (such as the Agency for Healthcare Research and Quality's Clinical Classifications) to the data set, and finally the third data set augmented the previous by adding binary variables providing birthing information. The statistical method of $K$-means and the machine learning algorithm Kohonen self-organizing maps then discovered between four to six patient clusters (depending on the stage of data preparation). Essentially the authors assigned these flow paths to a patient diagnosis.

The above studies indicate that, while classifying and clustering patient processes as a means to model flow have been adequate for situations in which patients are of a similar diagnosis (e.g. obstetrics) or in one unit (e.g. emergency department), it has not yet proved suitable for a vast range of patients across multiple hospital units. These approaches do not completely portray the complexity and dynamics of hospital resource allocation decisions. The review of literature also illustrates the lack of an adequate data source capturing patient-diagnosis-specific and organization-wide patient flow.

\section{DATA}

The literature review discussed the undeniable applicability of simulation research in hospital operations analysis. To further advance simulation analysis of hospital operations, simulation models need to have a more realistic representation of patient mix and the interdependence of hospital resources. Unfortunately, a suitable input data source has not yet been identified which provides this type of information. This section advances research of input data for hospital simulations by discussing how information system messages can be used to reconstruct patient flow patterns. It begins with a formal definition of patient flow and defines how patients are grouped by diagnosis. The section continues with an overview of HL7-formatted hospital information system messages and concludes by describing the five steps used to build a data mart of flow information.

\subsection{Definition of Patient Flow}

For the purposes of this tutorial, patient flow is defined as encompassing the following four concepts:

1. The set of events during a patient's stay;

2. The precedence/sequence of events;

3. The duration of events;

4. Resources required performing these events.

\subsection{Grouping of Patient Diagnoses}

The well-established Diagnosis Related Group (DRG) system is used to define patient types. This classification system provides both a clinical and operational perspective of patient activity. (Fetter 1991) The purpose of the DRG system is to relate types of patients treated to the resources they consume by classifying hospital cases into one of approximately 500 groups, expected to have similar hospital resource use. (Fetter 1991) A DRG is assigned to a patient by a "grouper" program based on ICD diagnoses, procedures, age, sex, and the presence of complications or comorbidities. Patients within each DRG are similar clinically and are expected to use a comparable level of hospital resources. Originally developed for Medicare as part of the prospective payment system, DRGs have been used since 1983 to determine how much Medicare pays hospitals for inpatient care. Today DRGs are the basis for almost all hospital billing systems in the United States. Centers for Medicare and Medicaid Services is responsible for the maintenance and modifications of DRG definitions.

\subsection{The Data Source - HL7 Formatted Messages}

With patient type defined, we now turn to the topic of comprehensive and electronically available data sources. For each patient admitted to a hospital, departmental information systems generate a sequence of messages that post medication orders, lab requests, transfer and transportation orders, and so forth. Vast amounts of health care information are exchanged on a daily basis. The variety of clinical terminology makes it difficult to display this information in a readable and concise manner. 


\section{Konrad and Lawley}

To ensure universal comprehension, messaging information needs to be presented in a standardized format and thus the HL7 protocol was developed. The messaging standard is widely used and enables disparate healthcare applications to exchange sets of clinical and administrative data (Health Level Seven n.d.) regardless whether the institution has electronic medical records. This research capitalizes on the wealth of information contained in these messages.

\subsection{Overview of HL7 Formatted Messages}

HL7 formatted messages are stored in patient trace logs capturing unique, time-stamped information regarding the services a patient undergoes while in the hospital. Each message is composed of a combination of segments. Each segment is composed of fields delimited by the character 'I'. Every field has its own unique purpose defined by the HL7 standard. An HL7 message is perhaps best illustrated through an example as shown in Figure 1, which contains a hypothetical patient admission message. The first segment, labeled MSH, contains information about the message itself such as the sending and receiving systems (Admission and Lab respectively), the type of the message, and so forth. The second segment, labeled EVN, denotes the message type, an admit (A01). The third segment, labeled PID, contains patient demographics while the NK1 includes next of kin data, and the PV1 segment lists patient visit information.

The example message tells us:

Patient William A. Jones, III was admitted on July 18, 1988 at 11:23 a.m. by doctor Sidney J. Lebauer (\#004777) for surgery (SUR). He has been assigned to room 2012, bed 01 on nursing unit 2000. His next of kin is his wife Barbara. The message was sent from system ADT (Admission) at the MCM site to system LABADT (Lab), also at the MCM site, on the same date as the admission took place, but three minutes after the admit.

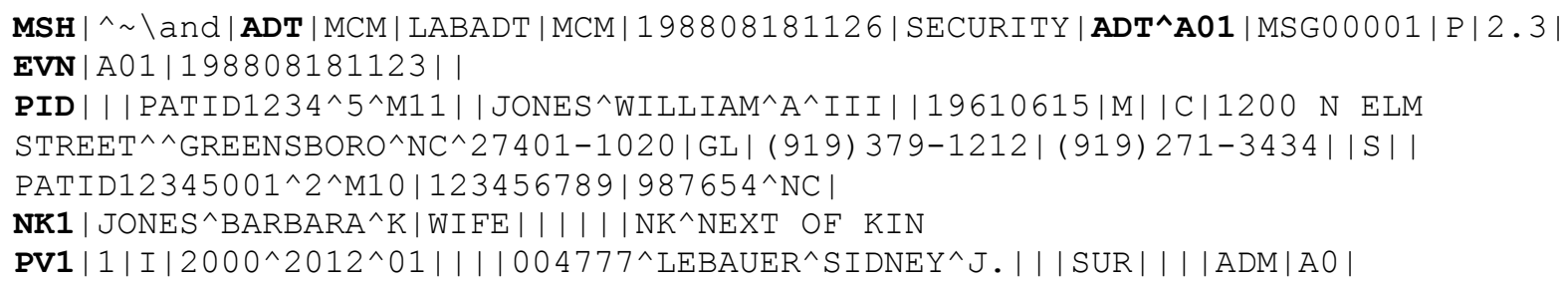

Figure 1: Sample HL7 message (Health Level Seven n.d.)

The set of HL7 formatted messages generated for a given patient represents much of what happens to the patient during their stay and contain a rich source of inpatient visit information. Details regarding the time, order status, ordering physician, and results for labs and diagnostic testing are present in these messages. Vital signs and procedure notes can also be found. In addition to clinical information, messages sent between information systems record bed transfers, patient location, meal changes, or admission time for a patient among a plethora of other information. Only a portion of the information found in these messages is required to reconstruct patient and resource flow paths. A patient's allergies, their insurance information and similar types of messages are ignored. The exact content of the data depends on a hospital's unique implementation. For example, segment codes beginning with the letter $\mathrm{Z}$ are reserved for locally-defined messages (examples include additional insurance information or additional patient arrival information). Health Level Seven specifies the structure of messages and their segments; however not all messages and segments are required in an implementation.

It is noteworthy that patient care paths for the individual patient are rarely formally recorded as such, they tend to evolve with the patient stay. Generally, patient care paths exist in a piece-meal fashion in the minds of physicians, nurses, and discharge planners. The information contained in these HL7-formatted messages presents an opportunity to reconstruct a patient path and make the patient path explicit.

\subsection{Overview of Data Preparation Steps}

The general approach for converting raw health information data into path data suitable for analysis is summarized in Table 1 , and each step is then described. A database was created to store this information. 


\section{Konrad and Lawley}

Table 1: Overview of data preparation steps

\begin{tabular}{|l|l|}
\hline Processing Step & Description \\
\hline 1. Removal of demographic information & $\begin{array}{l}\text { Remove patient identifiers and unnecessary message segments such as } \\
\text { those pertaining to insurance }\end{array}$ \\
\hline 2. Visit summary & Create a record with admission and discharge information for a patient \\
\hline 3. Visit details & Create a record for each message in a patient's trace log \\
\hline 4. Consolidation & $\begin{array}{l}\text { Delete unnecessary records (such as patient allergy information) and } \\
\text { consolidate duplicate records }\end{array}$ \\
\hline $\begin{array}{l}\text { 5. Match patient activity and resource re- } \\
\text { quirement }\end{array}$ & $\begin{array}{l}\text { Define resource requirements and assign resources required for each } \\
\text { activity during the patient visit }\end{array}$ \\
\hline 6. Patient sequences & Create a readable representation of an individual patient path \\
\hline
\end{tabular}

\section{Step 1 Removal of Patient Demographic Information}

Initially all data is received in text files; however, not all data is relevant for patient flow analysis. Message segments regarding patient demographics (PID, PD1, NK1); insurance (GT1, IN1, IN2); accidents (ACC), allergies (AL1) and customized "catch all" segments (ZPI, ZIN) are removed. Message classes regarding pharmacy administration (RAS, RGV, RDE); billing (BAR, DFT) and medical document management (MDM) were also removed. To further ensure patient anonymity all A08 (update patient information) messages were removed. Consults with nursing and patient flow researchers deemed these segments were not crucial to the reconstruction of patient flow.

\section{Step 2 Visit summary}

A summary record of every patient stay is created containing admission and discharge times, initial diagnosis, a patient's admission location, and a calculated length of stay.

\section{Step 3 Visit details}

Using the results of Step 2, a patient_activity_history table is created. Table 2 contains sample entries listing descriptions of each patient activity: a calculated field "time since admission" for the activity, the status of the order (e.g. SCScheduled, IP-in Process, CM- Completed), and patient location. This step seeks two types of information from the patient encounter: (1) admission/discharge (ADT) messages and (2) order messages. Appendix A contains ADT segments and classes of interest which refer to patient transfers, admission, registration, and discharge activities. On the other hand, order messages encompass any medical or clinical procedure undertaken during a patient's stay: diagnostic tests, meals, therapy, and so forth. Order information is available in ORM^ 001 type messages. Within the ORM^ $\mathrm{OR}^{\wedge} 1$ message, the ORC (Common Order) and OBR (Observation Request) segments are the primary sources of information.

Table 2: Sample records in the patient_activity_history table

\begin{tabular}{|c|c|c|c|c|c|c|}
\hline Patient & $\begin{array}{c}\text { Message } \\
\text { Type }\end{array}$ & $\begin{array}{c}\text { Time Since } \\
\text { Admission } \\
\text { (hours) }\end{array}$ & Activity & $\begin{array}{c}\text { Order } \\
\text { Status }\end{array}$ & $\begin{array}{c}\text { Order } \\
\text { Number }\end{array}$ & Location \\
\hline 1 & $\mathrm{ADT}^{\wedge} \mathrm{A} 01$ & 0.00 & Admit & & & S5M \\
\hline 1 & $\mathrm{ORM}^{\wedge} \mathrm{O} 01$ & 1.10 & Basic Metabolic Panel & SC & 314567 & SR5M \\
\hline 1 & $\mathrm{ORM}^{\wedge} \mathrm{O} 01$ & 1.75 & Complete Blood Count & SC & 313378 & SR5M \\
\hline 1 & $\mathrm{ORM}^{\wedge} \mathrm{O} 01$ & 7.50 & Basic Metabolic Panel & IP & 314567 & S5M \\
\hline 1 & $\mathrm{ORM}^{\wedge} \mathrm{O} 01$ & 8.10 & Complete Blood Count & CM & 316289 & S5M \\
\hline
\end{tabular}

\section{Step 4 Consolidation}

Order segments contain a wealth of order information including when an order is placed, when it is scheduled to take place, and when it has been completed. Correspondingly, a fair amount of data redundancy occurs in the patient_activity_history table. Typically each activity will have several rows pertaining to messages of various order states: scheduled (SC), in process (IP), completed (CM), etc. (See Order Number 314657 in Table 2.) 


\section{Konrad and Lawley}

For the purposes of patient path reconstruction, two streams of timing information are sought:

(1) The sequence of activities;

(2) The duration of an activity.

\section{Activity Sequence}

We recognize that two (or more) patient activities can happen in parallel. For example, a lab specimen may be processed while a patient is having an EKG. To establish the sequence of activities, the start time of the activity is obtained. In the afore mentioned example, the lab specimen would be drawn before the EKG, therefore the sequence would read: $\{$ LabTest, EKG\}. Determining the start time of an activity required analysis of the HL7 message as discussed in the next subsection.

\section{Activity Duration}

As discussed above, to establish the sequence of activities, the start time of the activity is required. Because resource utilization is of interest, the time when that activity ended is also needed. With start and end times for an activity, we can establish the duration of a particular activity (e.g. how long resources were used to complete a specific activity). Two pieces of information are necessary to establish activity start and end times: (1) the timestamp and 2) the order status (e.g. activity in process, activity ended). As illustrated in Table 3, a single activity such as a Basic Metabolic Panel could have multiple messages regarding the progression of the order. Even within a message describing a specific order status (e.g. 'Scheduled'), differing time information can exist. The two subsections below detail how time and status information were extracted for each order.

\section{1. $\underline{\text { Time }}$}

In consultation with HL7 experts at the study hospital and by following the guidelines in (Health Level Seven, 1997) the following three rules were developed to establish the start and end times of an activity.

Time Extraction Rule \#1: For procedures and radiology orders (e.g Cath Lab, X-ray). OBX segments of an ORU^R01 message can be used. If these fields are not filled, or the start time is the same as the stop time, use Rule \#2.

Time Extraction Rule \#2: For lab orders, diagnostic tests, transfusions, ventilators, and case management. If both ORC and OBR segments are present, the OBR values override the ORC values. For example, in Table 3, in Message \#6, the end time of the lab test would be judged to be 13:54.

Time Extraction Rule \#3: For admission/discharge/transfer activities. The EVN-6 field will be used. If this field is not filled the EVN-2 field can be used.

\section{2. $\quad$ Order Status}

There are two methods of obtaining order status information. If the status is that of the entire order, we use ORC-15, order effective date/time field and ORC-5, order status field. If the status pertains to the portion of the order detail segment (e.g. multiple administrations of the same medication), use OBR-25, result status and OBR-22, results report/status change date/time. If both the respective ORC and OBR fields are filled, the OBR values override the ORC values. Chapter 4 of (Health Level Seven, 1997) contains a description of all possible values for these two fields. It is important to mention that orders can be cancelled and thus need to be removed.

By identifying the correct timestamp and order status for each message, the start time and end time of an activity can be identified. Activities related to admission/discharge/transfer typically only had one associated message. 


\section{Konrad and Lawley}

Table 3: Example of messages for a laboratory test order

\begin{tabular}{|c|c|c|c|c|c|c|}
\hline $\begin{array}{c}\text { Message } \\
\quad \#\end{array}$ & $\begin{array}{l}\text { Order } \\
\text { Number }\end{array}$ & Description & $\begin{array}{l}\text { ORC-5 Order } \\
\text { Status } \\
\text { Value }\end{array}$ & $\begin{array}{l}\text { OBR-25 Result } \\
\text { Status } \\
\text { Value }\end{array}$ & $\begin{array}{c}\text { ORC-15 } \\
\text { Order } \\
\text { Effective } \\
\text { Date/ } \\
\text { Time/ } \\
\text { Value } \\
\end{array}$ & $\begin{array}{c}\text { OBR-22 } \\
\text { Results } \\
\text { Report/ } \\
\text { Status } \\
\text { Change }\end{array}$ \\
\hline 1 & 456123 & $\begin{array}{l}\text { Order was } \\
\text { scheduled }\end{array}$ & $\begin{array}{c}\text { SC } \\
\text { (Scheduled) }\end{array}$ & None & $9: 15$ & None \\
\hline 2 & 456123 & New order & $\mathrm{N}(\mathrm{New})$ & $\mathrm{N}(\mathrm{New})$ & $9: 27$ & $9: 33$ \\
\hline 3 & 456123 & $\begin{array}{l}\text { Order received, } \\
\text { specimen not } \\
\text { yet received }\end{array}$ & IP (In Process) & $\begin{array}{l}\text { O (Order received; specimen } \\
\text { not yet received) }\end{array}$ & $10: 59$ & $10: 59$ \\
\hline 4 & 456123 & $\begin{array}{l}\text { Specimen } \\
\text { received }\end{array}$ & IP(In Process) & $\begin{array}{l}\text { I (No results available; spe- } \\
\text { cimen received, procedure } \\
\text { incomplete) }\end{array}$ & $13: 17$ & $13: 10$ \\
\hline 5 & 456123 & $\begin{array}{l}\text { Preliminary } \\
\text { results }\end{array}$ & IP(In Process) & $\begin{array}{c}\text { P (Preliminary: a } \\
\text { verified early result is availa- } \\
\text { ble, final results not yet } \\
\text { obtained) }\end{array}$ & $13: 46$ & $13: 46$ \\
\hline 6 & 456123 & $\begin{array}{l}\text { Completed, } \\
\text { final results } \\
\text { stored and } \\
\text { verified } \\
\end{array}$ & $\begin{array}{c}\mathrm{CM} \\
\text { (Completed) }\end{array}$ & $\begin{array}{c}\text { F (Final results; results stored } \\
\text { and verified) }\end{array}$ & $14: 02$ & $13: 54$ \\
\hline
\end{tabular}

\section{Step 5 Integration}

With a concise list of patient activities and their start and end times, the next step in data preparation is to associate these activities to resources. Nursing researchers at Purdue University helped create a comprehensive matrix pairing activities with the resources required to complete them. A sample of this matrix is shown in Table 4. Both clinical and administrative patient activities were matched with the resources required to complete the task. For example an "Admit" activity requires a clerk, while a "Basic Metabolic Panel" requires a nurse to draw blood and a lab technician (denoted as Lab in Table 4) to analyze the sample. To avoid an overly intricate model, resources associated with an activity are assumed to work in parallel, not in sequence. For example, the activity "Basic Metabolic Panel," the process of a nurse drawing blood and then the lab analyzing it will not be modeled separately, but rather the nurse and lab are assumed to act in parallel.

Table 4: Sample of the matrix pairing patient activities with the required resources

\begin{tabular}{|l|c|c|c|c|c|c|c|}
\hline & \multicolumn{7}{|c|}{ Required Resource } \\
\hline Visit Activity & Nurse & Tech & Lab & Clerk & Physician & Radiology & Procedure \\
\hline $\begin{array}{l}\text { Arterial Blood } \\
\text { Gas }\end{array}$ & & $\mathrm{X}$ & & & $\mathrm{X}$ & & \\
\hline Admit & & & & $\mathrm{X}$ & & & \\
\hline $\begin{array}{l}\text { Basic Metabolic } \\
\text { Panel }\end{array}$ & $\mathrm{X}$ & & $\mathrm{X}$ & & & & \\
\hline Heart Cath & $\mathrm{X}$ & $\mathrm{X}$ & & & $\mathrm{X}$ & & $\mathrm{X}$ \\
\hline
\end{tabular}

A representative number for each combination of resources is then assigned, to which we refer as the Resource Bundle. For example, Resource Bundle 6 denotes a patient activity requiring a nurse and the lab (such as a Basic Metabolic Panel). Resource Bundle 1 represents activities requiring a clerk, such as patient registration. These bundles are listed in Appendix B. Note that another DRG may use different resources or a different combination of resources. The information from Table 3 and the table in Appendix A are combined to produce a time-ordered table listing activities a patient underwent along with the group of resources consumed during the activity. Table 5 contains a sample of this table. 


\section{Konrad and Lawley}

Table 5: Sample patient path with associated Resource Bundles

\begin{tabular}{|c|c|c|c|c|}
\hline Patient Number & Time since admission & Activity & Resources & $\begin{array}{c}\text { Resource } \\
\text { Bundle }\end{array}$ \\
\hline DRG558_9 & 0.00 & Admit & Clerk & 1 \\
\hline DRG558_9 & 0.63 & Culture, MRSA Screen & RN, Lab & 6 \\
\hline DRG558_9 & 1.15 & Basic Metabolic Panel & RN, Lab & 6 \\
\hline DRG558_9 & 1.46 & Complete Blood Count & RN, Lab & 6 \\
\hline DRG558_9 & 13.17 & 12 Lead EKG & Tech, MD & 7 \\
\hline DRG558_9 & 14.27 & Heart Cath & RN, Tech, MD & 15 \\
\hline DRG558_9 & 16.86 & 12Lead EKG & Tech, MD & 7 \\
\hline DRG558_9 & 20.18 & Troponin I & RN, Lab & 6 \\
\hline DRG558_9 & 23.32 & Basic Metabolic Panel & RN, Lab & 6 \\
\hline DRG558_9 & 28.82 & Discharge & Clerk & 1 \\
\hline
\end{tabular}

This concept of representing flow from the perspective of workload requirements represents a novel approach of describing patient flow.

\section{Step 6 Patient sequences}

Visualizing and analyzing patient paths through a hospital is somewhat difficult in a table format such as Table 5, as each visit spans multiple records. To remedy this, a data construct called the patient sequence was developed. This construct shows the sequence of resource bundles used by a patient during his/her visit. For each patient, the resource bundle fields are consolidated in a time-ordered string with a * delimiter. A few of these consolidated records appear in Table 6 below.

Table 6: Sample patent sequences for DRG 558

\begin{tabular}{|l|l|}
\hline Patient ID & Path \\
\hline DRG558_1 & $* 1 * 14 * 7 * 6 * 6 * 6 * 6 * 10 * 6 * 14 * 6 * 6 * 6 * 6 * 6 * 6 * 6 * 6 * 6 * 6 * 6 * 7 * 15 * 10 * 14 * 7 * 6 * 6 * 6 * 7 * 1$ \\
\hline DRG558_2 & $* 1 * 14 * 7 * 6 * 6 * 6 * 6 * 15 * 6 * 6 * 7 * 7 * 6 * 6 * 6 * 6 * 6 * 1$ \\
\hline DRG558_3 & $* 1 * 14 * 7 * 10 * 6 * 6 * 6 * 6 * 6 * 7 * 14 * 6 * 7 * 15 * 6 * 6 * 6 * 7 * 1$ \\
\hline DRG558_7 & $* 1 * 14 * 6 * 6 * 6 * 14 * 6 * 6 * 6 * 7 * 6 * 6 * 14 * 15 * 6 * 6 * 7 * 7 * 1$ \\
\hline
\end{tabular}

\section{DIAGNOSIS SPECIFIC FLOW MODELS}

The previous section depicts how to obtain a sequence of resources a single patient uses. In this section, we synthesize a set of individual sequences into a representative model of patient flow for a given DRG.

The variability of paths taken through the hospital, even within a strictly defined DRG grouping, poses a significant modeling challenge. Within our small initial samples of approximately 25 patients for a DRG, each patient generated a unique path. Each patient sequence can be thought of as an instantiation of an underlying event generating process. Thus, a set of sequences can be likened to a distribution needing to be fit. In simplest terms, a distribution is a compact representation that captures the central tendency and variability in the data. At this point in the tutorial, we fit a flow model to the set of sequences. The flow model is a representation capable of capturing the main structural features of the sequences while also accounting for their variability. Structural features of interest are resources, frequently occurring subsequences, and timing.

A flow model serves three related purposes in a simulation: the generation, description, and analysis of patient flow sequences. As with a statistical distribution, a flow model can produce an instance of a population- a sequence of resources a patient uses throughout their entire hospital visit. A patient flow model is descriptive. It allows the resources used by a diagnosis to be summarized numerically. Basic examples of numerical descriptors include the frequency of resource usage or the 


\section{Konrad and Lawley}

mean length of stay. Furthermore, because flow sequences are modeled in a way that accounts for randomness and uncertainty in the observations, a flow model can be used to draw inferences about a about a diagnosis.

Patient flow models are represented as both Petri nets and regular expressions. Both formats can be used as simulation input as described in Section 5. A regular expression model can easily generate valid patient paths. A Petri net model can be coupled with a hospital simulation model for detailed operational analysis.

Regular expressions are a powerful construct used for representing complex patterns in structured strings. (Freidl 2002) A regular expression is a special text string for describing a pattern according to syntax rules. These constructs are frequently used in string searches, perhaps the most familiar regular expression being the "**" character used in file searches. A regular expression pattern defines a grammar. All words (or in our case, patient sequences) which match the pattern are said to be in the language defined by the grammar. A regular expression grammar language dictionary generator can generate all possible strings that match a given pattern. (Friedl 2002) Using a regular expression, a simulation modeler can generate hundreds of possible patient paths for a given DRG, provided the underlying regular expression model for the DRG is correct.

Petri nets are a graphical and mathematical modeling method well suited to describe and study systems characterized as being concurrent, asynchronous, distributed, parallel and/or stochastic. (Murata 1989) Petri nets capture how the system state evolves and identifies how loads tend to propagate from one unit to another. It is also possible to incorporate timing information into a Petri net model and thus describe the evolution of system behavior. (Murata 1989; Peterson 1981) Petri nets also support modularity and abstraction. Using a Petri net representation of patient flow allows a modeler to couple diagnosisspecific data of resource utilization with a hospital operations simulation model.

A close relationship exists between regular expressions and Petri nets. In a Petri net, events are modeled by transitions, and the occurrence of an action is modeled by transition firings. Sequences of events are modeled by sequences of transitions. Thus, the set of allowable transition sequences characterizes a Petri net and (to the degree that the Petri net correctly models a system) the modeled system. (Peterson 1981) Baker (1972) introduced the basic idea of associating a language with a Petri net and using this language to describe the behavior of the net. The set of strings generated by all possible firing sequences defines a formal language called a Petri net language. [See Peterson (1981) for a survey of Petri net languages.] A Petri net language can be expressed as a regular language. (Peterson 1981) Every regular language can be obtained from a regular expression. Conversely, with every regular expression, we can associate a regular language. (Kleene 1956)

\subsection{Flow Model Development}

The previous section discussed why individual patient sequences, obtained from HL7 trace logs, should be amalgamated by diagnosis. Both regular expressions and Petri nets provide a suitable format to capture patient flow by diagnosis, each having its own merits. Regular expressions can generate patient sequence input data for a simulation model. The modular aspect of Petri nets allows diagnosis-specific, multi-departmental resource utilization, information to be introduced into a simulation. This information renders a more realistic portrayal of hospital functions and provides a greater confidence in model results. In this section we discuss how these flow models were developed. For illustrative purposes, we use DRG 558- Percutaneous Cardiovascular Procedures with Drug-Eluting Stent without Major Complications.

Both regular expression and Petri net models were constructed by inspecting the transition probability matrix and individual patient sequences. The transition probability matrix establishes which events can follow another event, and the patient sequences prescribe the event order. For example, DRG 558's transition matrix indicates that a bed transfer (Resource Bundle 14) can precede any event in DRG 558 sequences. However, upon examining all the sequences, it is obvious that a sequence beginning with the subsequence $\{14,1\}$ is inadmissible (a bed transfer followed by an admit event); however, the same subsequence can be found towards the end of the sequence (bed transfer followed by discharge). For this reason, inspection of both the transition probability matrix and actual sequences is necessary to generate a flow model for a given DRG.

\subsection{Regular Expression Models}

Figure 2 contains the regular expression model for DRG 558. Each symbol in the expression corresponds to a Resource Bundle. For example, a "1" in the regular expression corresponds to a Resource Bundle 1 (use of the clerk for admit/discharge processes). The + operator in the regular expression states that a patient sequence must contain at least one of the events preceding it. For example, in Figure 2, a sequence must contain at least one of Radiology Events (subsequence beginning with the symbol "7"), EKG (subsequence beginning with the "10" symbol), bed transfer (subsequence beginning with the "14" symbol). The ? operator indicates one item allowed, but it is optional. Friedl (2002) is an excellent reference on regular expressions. 


\section{$114(14) ?(7|10| 14)+15(7|10| 14)+1$}

Figure 2: The regular expression model for DRG 558

Figure 3 contains the reduced Petri net model for DRG 558. In a Petri net places are depicted as circles, and transitions are represented as bars. When modeling a system, transitions represent events (such as a patient being discharge), and the necessary inputs for the event are modeled as places (such as a nurse). The main features of the DRG 558 model are summarized in Konrad (2009).

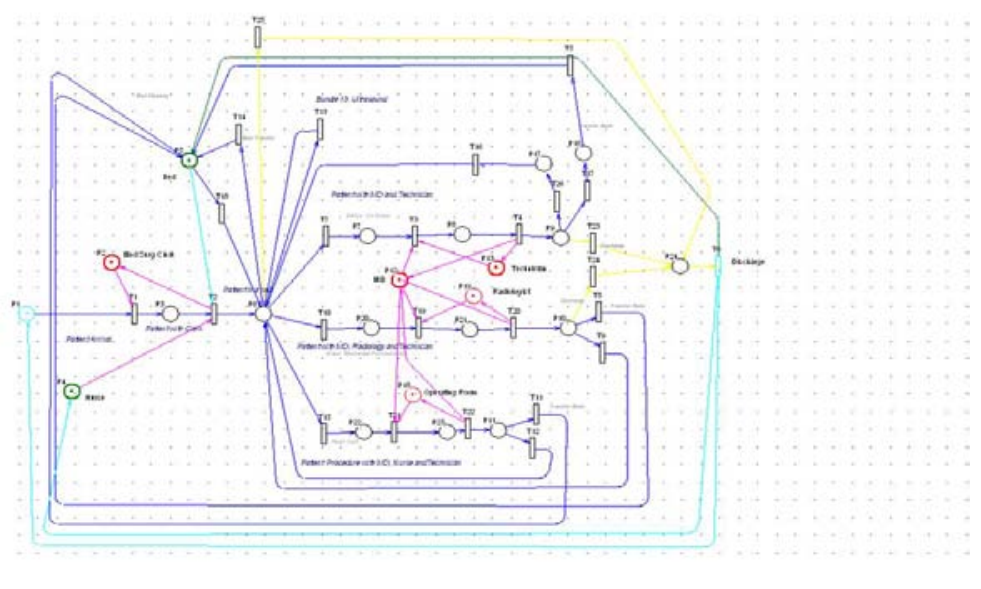

Figure 3: The Petri net model for DRG 558

To authenticate that our methods reflect actual patient movement, we compared and contrasted the HL7-generated patient sequences against patient charts. This validation exercise affirmed that information system generated messages reasonably reflect resource usage and inpatient flow in a hospital. (See Konrad (2009) for details.) We then established the correctness of both the Petri net and regular expression models through formal proofs. (See Konrad (2009) for details.) The verification exercise identified a small number of structural errors in both the regular expression and Petri net models. These errors were subsequently corrected. In the subsequent section, we illustrate how these flow models can be applied in hospital simulation modeling.

\section{FUTURE APPLICATIONS}

As described in Section 2, a multi-departmental analysis which considers a hospital's patient mix provides planners with more realistic results. The method presented in Sections 3 and 4 develop an inpatient flow model from information system messages. This approach creates a comprehensive and unrealized source of simulation input capable of capturing the resource loading imposed across a hospital by a patient's diagnosis. This section advances the discussion by shifting the focus from the methodological to the applied domain. The inpatient flow models open a plethora of possibilities to learn about actual hospital operations. Given that the flow models developed represent actual inpatient resource utilization in a hospital, there are several possible applications. We briefly discuss one such application- analyzing short-term operational policies such as when to issue an ambulance divert or how to prioritize housekeeping.

Determining when to enact, or when to revoke, a short-term policy requires the integration of multiple sources of information. In this situation, the decision maker is interested in the transient behavior of a system, as opposed to the steady state. In this type of analysis, a decision maker seeks information such as current resource capacity, anticipated incoming workload from upstream departments, and the capacity of downstream departments. The hospital's mix of diagnoses will dictate how resource requirements will change over the short-term. For example, if the hospital currently has a high volume of joint replacement patients, a planner can anticipate low lab and ICU utilization and high physical therapy utilization. All this information must be amalgamated, and a decision maker must assess the implications of executing or reversing a policy. 


\section{Konrad and Lawley}

A recent study (Varadarajan 2008) and current research are laying the foundation for this type of analysis. The product of these studies is the Virtual Hospital Model (VHM). The Virtual Hospital Model is a generic representation of a hospital developed in consultation with hospital administrators, hospital staff, and health researchers. This model serves as a test bed for analyzing for both short-term and long-term operational policies. The VHM consists of the following departments: The Emergency Department, the Operating Room, Medical Surgical Department, the Intensive Care Unit, the Radiology Department and a centralized Lab.

The VHM capitalizes on the properties of Petri nets. Individual hospital units and processes are developed as individual nets. These nets can be joined at common nodes, such as the place node representing radiology staff. A net replication program automatically transforms a Petri net model into an Automod simulation. A modeler can customize their model (e.g. housekeeping is centralized vs. dedicated) or expand certain portions of the hospital net (e.g. differentiate between cardiac, neurological, pediatric ICUs) by making changes in the Petri net(s). The Petri net replication program automatically generates the customized or revised simulation model. The VHM can be thought of as a skeleton of hospital operations and dictates the intra- and interdepartmental relations. However, the evolution of the state of the hospital is governed by the patient load. Thus the diagnosis-specific nets described in the previous section are joined with the VHM. Current research is investigating the relationship between the current workload in a hospital and the likelihood of reaching a divert state.

\section{SUMMARY}

This tutorial paper highlights the difficulties encountered in simulation hospital models, namely conducting a multidepartmental analysis and accounting for a hospital's patient mix. Researchers advocate using a patient flow approach to analyze hospital operations, yet the data to do so is lacking. This tutorial addresses these issues by reconstructing actual patient flow paths from HL7-formatted communication exchanges. Individual flow paths for a given diagnosis are synthesized into models suitable for simulation studies- regular expressions and Petri nets. Finally we discuss how they type of input data can be used to examine short-term operational decisions in a hospital.

\section{A HL7 MESSAGE TYPES AND SEGMENTS USED}

\begin{tabular}{|l|l|}
\hline Message Types & $\mathrm{ADT}^{\wedge} \mathrm{A} 01, \mathrm{ADT}^{\wedge} \mathrm{A} 03, \mathrm{ADT}^{\wedge} \mathrm{A} 04, \mathrm{ADT}^{\wedge} \mathrm{A} 06, \mathrm{ORM}^{\wedge} \mathrm{O} 01, \mathrm{ORU}^{\wedge} \mathrm{R} 01$ \\
\hline Message Segments & $\mathrm{EVN}, \mathrm{MSH}, \mathrm{ORC}, \mathrm{OBR}, \mathrm{OBX}, \mathrm{PVI}$ \\
\hline
\end{tabular}

\section{B RESOURCE BUNDLES}

Resource Bundles for DRG 558 patients

\begin{tabular}{|l|l|l|}
\hline $\begin{array}{l}\text { Resource } \\
\text { Bundle }\end{array}$ & Description & Examples \\
\hline 1 & Clerk & Admit, Discharge \\
\hline 6 & Nurse and Lab & Lab tests- need to draw specimen and analyze results \\
\hline 7 & Tech and MD & Echocardiogram (EKG) \\
\hline 10 & Tech, MD, and Radiology & X-ray, Computed Tomography (CT) Scan with contrast \\
\hline 13 & Ultrasound Tech, and MD & Aorta Ultrasound \\
\hline 14 & Bed Transfer & \\
\hline 15 & Procedure & Cardiac Catheterization \\
\hline
\end{tabular}

\section{REFERENCES}

Baesler, F., H. Jahnsen, and M. DaCosta. 2003. The Use of Simulation and Design of Experiments for Estimating Maximum Capacity in An Emergency Room. In Proceedings of the 2003 Winter Simulation Conference, ed. S. Chick, P. J. Sánchez, D. Ferrin, and D. J. Morrice, 1903-1906. Piscataway, New Jersey: Institute of Electrical and Electronic Engineers, Inc.

Baker, H. 1972. Petri nets and languages. MIT, Computation Structures Group Memo 68, Project MAC, Cambridge, Massachusetts. 


\section{Konrad and Lawley}

Brahimi, M., and D. Worthington. 1991. Queueing models for out-patient appointment systems - a case study. Journal of the Operational Research Society 42: 733-746.

Cameron, P., P. Scown, and D.Campbell. 2002. Managing access block. Australian Health Review 25: 59-68.

Ceglowski, A., L. Churilov, and J. Wassertheil. 2005. Knowledge Discovery through Mining Emergency Department Data. In Proceedings of the 38th Hawaii International Conference on System Sciences, 3-6. Hawaii, USA: Institute of Electrical and Electronic Engineers, Inc.

Denton, B., A. Rahman, H. Nelson, et al. 2006. Simulation of a multiple operating room surgical suite. In Proceedings of the 2006 Winter Simulation Conference, ed. L. F. Perrone, F. P. Wieland, J. Liu, B. G. Lawson, D. M. Nicol, and R. M. Fujimoto, 414 - 424. Piscataway, New Jersey: Institute of Electrical and Electronic Engineers Inc.

DesRoches, C., E. Campbell, S. Rao, et al. 2008. Electronic health records in ambulatory care-a national survey of physicians. New England Journal of Medicine 359 (1): 50-60.

Fatovich, D., Y. Nagree, and P. Sprivulis. 2005. Access block causes emergency department overcrowding and ambulance diversion in Perth, Western Australia. Emergency Medicine Journal 22: 351-354.

Fetter, R. 1991. Diagnosis related groups: Understanding hospital performance. Interfaces 21 (1): 6-26.

Friedl, J. 2002. Mastering Regular Expressions. 2nd ed. Cambridge: O'Reilly.

Harper, P. 2002. A Framework for Operational Modeling of Hospital Resources. Health Care Management Science 5 (3): $165-73$.

Health Level Seven. n.d. Available via <http://www.h17.org> [ accessed May 29, 2008]

Health Level Seven. 1997. HL7 V2.3 Guide.

Hughes, M., E. Carson, M. Makhlouf, et al. 1998. A Petri Net Based Model of Patient-Flows in a Progressive Patient-Care System. In Proceedings of 20th Annual International Conference of the IEEE Engineering in Medicine and Biology Society, 3048-3051.

Hung, G., S. Whitehouse, C. O'Neill et al. 2007. Computer Modeling of Patient Flow in a Pediatric Emergency Department Using Discrete Event Simulation. Pediatric Emergency Care 23 (1): 5-10.

Isken, M. 2002. Modeling and Analysis of Occupancy Data: A Healthcare Capacity Planning Application. International Journal of Information Technology and Decision Making 1 (4): 707-729.

Isken, M., V. Sugumaran, T. Ward, et al.2005. Collection and Preparation of Sensor Network Data to Support Modeling and Analysis of Outpatient Clinics. Health Care Management Science 8 (2):87-99.

Jacobson, S., S. Hall, and J. Swisher. 2006. Discrete-Event Simulation of Health Care Systems. In R. W. Hall, Patient Flow: Reducing Delay in Healthcare Delivery, 211-252. Springer International Series.

Jun, J., S. Jacobson, and J. Swisher. 1999. Application of discrete-event simulation in health care clinics: A survey. Journal of the Operational Research Society 2: 109-123.

Klassen, K., and T. Rohleder. 1996. Scheduling outpatient appointments in a dynamic environment. Journal of Operations Management 14: 83-101.

Kleene, S. 1956. Representation of events in nerve nets and finite automata. Automata Studies.

Kolker, A. 2008. Process Modeling of Emergency Department Patient Flow: Effect of Patient Length of Stay on ED Diversion. Journal of Medical Systems 32 (5): 389-401.

Komashie, A., A. Mousavi, and J. Gore. 2008. Using Discrete Event Simulation (DES) to Manage Theatre Operations in Healthcare: An Audit-Based Case Study. In UKSIM 2008 Tenth International Conference on Computer Modeling and Simulation, 360-365. Cambridge, UK.

Konrad, R. 2009. Inpatient Flow from Hospital Information Systems. Ph.D. thesis, School of Industrial Engineering, Purdue University, West Lafayette Indiana.

Ledersnaider, D., and B. Channon. 1998. SDM95-reducing aggregate care team costs through optimal patient placement. Journal of Nursing Administration 28: 48-54.

Lin, F., L. Hsieh, and S. Pan. 2005. Learning Clinical Pathway Patterns by Hidden Markov Model. In Proceedings of the 38th Hawaii International Conference on System Sciences, 142a. Hawaii, USA: Institute of Electrical and Electronic Engineers, Inc.

Mackay, M. 2001. Practical experience with bed occupancy management and planning systems: an Australian view. Healthcare Management Science 4: 47-56.

Marshall, A., C. Vasilakis, and E. El-Darzi. 2005. Length of Stay-Based Patient Flow Models: Recent Developments and Future Directions. Health Care Management Science 8(3): 213-220.

Murata, T. 1989. Petri nets: Properties, analysis and applications. Proceedings of the IEEE 77 (4): 541-580.

Oddoye, J., D. Jones, M. Tamiz, et al. 2009. Combining simulation and goal programming for healthcare planning in a medical assessment unit. European Journal of Operational Research 193 (1): 250-261.

Peterson, J. 1981. Petri Net Theory and the Modeling of Systems. New Jersey: Prentice-Hall. 


\section{Konrad and Lawley}

Rajagopalan, B., and M. Isken. 2001. Exploiting data preparation to enhance mining and knowledge discovery. IEEE Transactions on Systems, Man and Cybernetics 31 (4): 460-467.

Reid, P., Compton, W., Grossman, J., and Fanjiang, G. 2005. Building a Better Delivery System: a New Engineering/Health Care Partnership. National Academy of Engineering and Institute of Medicine, Committee on Engineering and the Health Care System. Washington DC: National Academy Press.

Schull, M.,K. Lazier, M. Vermeulen, S. Mawhinney, et al. 2003. Emergency department contributors to ambulance diverision: a quantitative analysis. Annals of Emergency Medicine 41: 467-476.

Sepulveda, J., W.Thompson, F. Baesler, et al. 1999. The use of simulation for process improvement in a cancer treatment center. In Proceedings of the 1999 Winter Simulation Conference, ed. P. A. Farrington, H. B. Nembhard, D. Sturrock, and J. Evans, 1541-1548. Piscataway, New Jersey: Institute of Electrical and Electronic Engineers, Inc.

Smith, P., and M. Goddard. 2002. Performance management and Operational Research: a marriage made in heaven? Journal of the Operational Research Society 53: 247-255.

Spry, C., and M. Lawley. 2005. Evaluating hospital pharmacy staffing and work scheduling using simulation. In Proceedings of the 1999 Winter Simulation Conference, ed. M. E. Kuhl, N. M. Steiger, F. B. Armstrong, and J. A. Joines, 2256 2263. Piscataway, New Jersey: Institute of Electrical and Electronic Engineers, Inc.

Swisher, J., and S. Jacobson. 2002. Evaluating the Design of a Family Practice Healthcare Clinic Using Discrete-Event Simulation. Health Care Management Science 5 (2): 75-88.

Takakuwa, S., and H. Shiozaki. 2004. Functional Analysis for Operating Emergency Department of a General Hospital. In Proceedings of the 2004 Winter Simulation Conference, ed. R .G. Ingalls, M. D. Rossetti, J. S. Smith, and B. A. Peters, 2003-2011. Piscataway, New Jersey: Institute of Electrical and Electronic Engineers, Inc.

VanBerkel, P., and J.Blake. 2007. A comprehensive simulation for wait time reduction and capacity planning applied in general surgery. Health Care Management Science 10 (4): 373-385.

Vanden Bosch, P., and D. Dietz. 2000. Minimizing expected waiting in a medical appointment system. IIE Transactions 32: 841-848.

Varadarajan, N. 2008. Emergency Department Modeling and Analysis using Petri Nets-Master of Science in Industrial Engineering. Purdue University, School of Industrial Engineering.

Vermeulen, I., S. Bohte, S. Elkhuizen, et al. 2009. Adaptive resource allocation for efficient patient scheduling. Artificial intelligence in Medicine 46 (1): 67-80.

Wijewickrama, A., and S. Takakuwa. 2006. Simulation Analysis of an Outpatient Department of Internal Medicine in a University Hospital. In Proceedings of the 2003 Winter Simulation Conference, ed. L. F. Perrone, F. P. Wieland, J. Liu, B. G. Lawson, D. M. Nicol, and R. M. Fujimoto, 425-432. Piscataway, New Jersey: Institute of Electrical and Electronic Engineers, Inc.

\section{AUTHOR BIOGRAPHIES}

RENATA KONRAD is an Assistant Professor in the Industrial Engineering Program in the Department of Management at Worcester Polytechnic Institute. She received her Ph.D. in Industrial Engineering at Purdue University. She received her B.A.Sc and M.A.Sc. in Industrial Engineering at the University of Toronto. She has held engineering and systems analysis positions at American Management Systems Inc (now CGI), Loblaw Companies Ltd., and Toronto East General Hospital. Her research interests span patient flow, Petri-nets and health informatics. Her email address is $\langle r k o n r a d @ w p i$. edu $\rangle$.

MARK LAWLEY is a Professor in the Weldon School of Biomedical Engineering at Purdue University. Before joining Biomedical Engineering in 2007, he served nine years as Assistant and Associate Professor of Industrial Engineering, also at Purdue, and two years as Assistant Professor of Industrial Engineering at the University of Alabama. He is particularly interested in developing optimal decision policies for system configuration and resource allocation in large healthcare systems. He has focused on research initiatives with Wishard Hospital, the Regenstrief Institute of Indianapolis, the Richard L. Roudebush Veterans Administration Medical Center, Ascension Health, St. Vincent Hospitals, and the American College of Physicians. His research has been supported by the National Science Foundation, Union Pacific Railroads, Consilium Software, General Motors, Ascension Health, the Indiana State Department of Health, the Regenstrief Foundation, the St. Vincent Ministry, and many others. He received the $\mathrm{PhD}$ in Mechanical Engineering from the University of Illinois at Urbana Champaign in 1995 and is a registered Professional Engineer in the State of Alabama. His email address is <malawley@purdue.edu>. 